\title{
Electromagnetic Enhanced Ignition
}

Amanda L. Higginbotham Duque ${ }^{\mathrm{a}}$, W. Lee Perry ${ }^{\mathrm{a}}$

${ }^{a}$ High Explosive Science and Technology, Los Alamos National Laboratory, PO Box 1663, MS C920, Los Alamos, NM 87545, United States

*corresponding author: aduque@ @lanl.gov, +1 (505) 665-3417

\begin{abstract}
Here, we investigate how EM radiation affects the thermal decomposition pathway in HMX. The experiment used an external heat source $\left(\mathrm{CO}_{2}\right.$ laser) to rapidly heat the surface of HMX and observe the response upon application of EM energy that, on its own, is not enough power to induce heating or ignition. We hypothesize that charged intermediate decomposition species and free radicals in the gas phase interact strongly with EM energy, leading to plasma formation. These gas phase species form as a result of HMX sublimation and decomposition, and will act as "virtual antennas" and strongly couple to EM energy. The rapid absorption of EM energy during this coupling event is observed in the measured reflected power data. Ignition and plasma formation were monitored using both visible and IR photodiode probes, as well as imaged using a high-speed video camera. These observations support the hypothesis that the presence of an EM field will perturb the thermal decomposition pathway of HMX, and cause ignition to occur at a lower temperature than what is predicted under typical thermal conditions. This intense interaction results in electrically excited molecules that propagate the energy and surpass the activation barrier for ignition before the predicted ignition temperature of the bulk sample has been reached. Understanding the decomposition of energetic materials under the influence of EM energy is important to understand and predict material response under a variety of environmental conditions.
\end{abstract}

\section{Keywords}

Explosives; plasma; electromagnetic energy; ignition; decomposition; microwaves

\section{Introduction}

The use of explosives and other energetic materials has a significant world wide economic, political and strategic impact. Ongoing research continues to develop a better fundamental understanding of energetic materials to support detection, defeat, safety and performance aspects of this class of material. Initiation is an important subset of this overall topic area. The canonical understanding of accidental or intentional initiation of any explosive or propellant shows that it occurs when a critical quantity of energy is deposited in a critical volume of material at a sufficient rate to overcome heat losses. Sources of heat include impact, spark, friction, shock, and boundary heat (e.g. fire). While spark initiation is technically electromagnetic (EM) in nature, the general effect of EM energy on initiation has only been sparsely investigated [1-6]. The motivation for understanding this effect comes from our 
increasing dependence on radar and wireless communication sources in environments where explosives and weapons are present. Additionally, this form of energy may be a useful, novel initiation source.

While long wavelength radiation can interact with energetic materials in a strictly 'linear' thermal manner to cause ignition, we know little about how EM radiation might interact with the fundamental physical and chemical decomposition processes that are intrinsic to the initiation process. As this interaction might lead to an interesting, novel and safety-relevant 'non-linear' response, our work reported here investigates the direct interaction of EM radiation with the thermal decomposition pathway in octahydro-1,3,5,7-tetranitro-1,3,5,7-tetrazocine (HMX), the energetic component of a number of plastic bonded explosive and rocket propellant formulations. This interaction might occur via the presence of charged intermediate species present in the gas phase during thermal decomposition, and indeed the established field of plasma-assisted combustion utilizes EM interactions for combustion enhancement in gases and has been shown to improve ignition characteristics and flame speeds [7,8]. These studies suggest that the enhancements and improvements occur via interaction of the electric field with free electrons present in the gas phase, which will then transfer additional non-thermal energy to the intermediate decomposition species. An external electric field will accelerate electrons; energy is transferred to the gas phase molecules by electron impact to the internal degrees of freedom. The increase in the number of excited states and the dissociation and ionization of the molecules will cause an increase in the system reactivity and facilitate ignition and flame propagation.[8]

In an earlier publication we presented a study that revealed ignition of HMX during microwave heating $[9,10]$ occurred at a lower than predicted temperature as was expected from standard ignition calculations, which are supported by a relatively large body of literature [1115]. That work was intended to utilize the high bulk heating rates available from microwave heating to generate ignition data to challenge kinetic models for HMX in a sparsely investigated ignition-time regime. During our experiments, we observed optical emission at low bulk temperatures, as well as a sharp drop in reflected power signal just before ignition occurs (this feature will be discussed in greater detail in the Results section) $[9,10]$. These observations, in conjunction with the low ignition temperature, led to the hypothesis that microwave heating caused volatilization of HMX that subsequently decomposed into gas phase intermediates[16] which would interact with the EM radiation. In this manner, the EM radiation added energy directly to the gas phase via the acceleration of the charged particles, ultimately leading to ignition. Figure 1 shows a high level overview of how the general multi-step thermal decomposition pathway of HMX[17] is disrupted by the introduction of EM energy. We hypothesize that once thermal decomposition intermediates are present in the gas phase (Step 3), the introduction of EM energy may effect the typical progression of Step 4 by providing additional non-thermal energy into the system. Free electrons and free radical species generated from the decomposition intermediate gas phase molecules during HMX dissociation (that are a natural part of many decomposition/dissociation reactions) would interact directly with the EM energy. Therefore, beyond Step 3 the mechanism would be perturbed, and consequently the mathematical description would be altered as well (i.e. temperature cannot be adequately described using the standard kinetic expression). More detail on the thermal decomposition mechanism of HMX and intermediate species that are formed may be found in [16] and [18]. Figure 1 also proposes potential chemical structures of the gas phase intermediate charged radical species, and their associated free electrons, that will act as "virtual antennas" and transfer non-thermal energy from application of the EM field into the system. This phenomenon is 
distinct from the observations for enhanced ignition and combustion,[7] where the interaction was presumably with charged combustion products, as opposed to decomposition intermediates (a subtle yet important difference). In the former case, either combustion was already occurring, or an external source created the conditions for ignition to occur.

Here, we examine this effect by utilizing a laser to heat the surface of a powder HMX sample, with synchronized EM pulses. These experiments provide additional evidence that support our hypothesis that EM radiation interacts preferentially to gas phase decomposition intermediate species and free electrons, and will ultimately alter the decomposition pathway of HMX typically observed under boundary thermal heating conditions.

\section{Experimental Details}

The overall schematic of the experimental setup is shown Figure 2.

\subsection{Sample Introduction}

Approximately 0.25 grams of fine HMX powder was hand-pressed in the center of a Teflon block sample holder (approximately 75\% TMD). The sample holder fills the inner volume of a WR-284 waveguide; the center of the Teflon block contains an opening for the sample, and is aligned with a 0.25 " diameter opening in the top of a straight piece of waveguide. The waveguide is terminated by a sliding short that was positioned to cause the peak of the electric field standing wave pattern to coincide with the sample location.

\subsection{Application of EM Energy}

A magnetron system provided pulsed power at a peak of $3 \mathrm{~kW}$ and frequency of 2.45 $\mathrm{GHz}$. Directional couplers crystal detectors (bolometers) provided voltage-proportional power signals to measure forward and reflected power incident on, and reflected from, the heating fixture.

\subsection{Laser Heating}

The laser used for surface heating of $\mathrm{HMX}$ was a $250 \mathrm{~W} \mathrm{CO}_{2}$ laser (Coherent model GEM-200PC) operating at a wavelength of $10.6 \mu \mathrm{m}$ and $50 \%$ power.

\subsection{Optical Diodes}

We used two silica fibers $(600 \mu \mathrm{m}$ diameter, NA $=0.32)$ coupled to two different detectors for measurement of heating and visible light generation; the tip of the fibers were held together and positioned just above the sample surface (non-contact). The IR detector (Thorlabs PDA10DT InGaAs, $1200-2570 \mathrm{~nm}$ ) provides good signal-to-noise above $80^{\circ} \mathrm{C}$. The visible light detector (Thorlabs DET210 silicon, 200-1100 nm) utilized a filter to eliminate IR wavelengths (Thorlabs FM01 near-IR hot mirror, transmission above $700 \mathrm{~nm}$ ) and was placed inline to ensure that all response measured was from the presence of visible light only.

It is important to note that we report the normalized visible and IR detector responses measured during the experiments. For the IR detector, a rise off the baseline implies a temperature above $80{ }^{\circ} \mathrm{C}$; the corresponding, calibrated absolute value temperatures are not reported. Instead, we use these detectors to probe a typical thermal versus plasma-type response. Similarly, the plasma responses are not correlated with temperature/brightness absolute values. 
The distinction between these two effects does not necessitate conversion to absolute temperatures.

\subsection{High Speed Video Imaging}

A Phantom Miro digital high speed camera was used, equipped with a Nikon ED lens (AF Micro Nikkor, 200mm). The capture resolution was 800x600, sample rate 1200 pps, exposure time $100 \mathrm{us}$, and total duration $=2.459 \mathrm{~s}$ (frame interval $=833 \mu \mathrm{s}$ ).

\section{Results}

Further analysis of data previously reported [10] revealed a feature in the reflected power signal (see highlighted regions in Figure 3) that represent fast absorption of power by the sample just before ignition occurs. This is seen as a sharp drop in the reflected power signal that deviates from the behavior elsewhere within the pulse; ignition may be detected in the reflected power by a sharp increase to nearly $100 \%$, or complete decoupling of the system. The total power absorbed is the reflected power subtracted from the forward power; thus increased power is absorbed when the reflected power is lower. We believe this represents the timing in which enhanced coupling between the EM energy and gas phase ions/radicals begins, leading to rapid absorption of EM energy causing subsequent ignition/plasma formation. This feature motivated the experiments described below; to further probe this behavior, we used an external heat source $\left(\mathrm{CO}_{2}\right.$ laser $)$ to rapidly heat the surface of HMX and observe the response upon application of EM energy that, on its own, is not enough power to induce heating or ignition. Although laser heating does not heat the sample in bulk, it does rapidly heat the surface and will induce sublimation at the sample-air interface - a criteria that we hypothesize is critical for plasma formation to occur. Laser ignition of HMX has been previously modeled[18,19] and studied experimentally[20] so the heating behavior of HMX under these conditions is well-understood. Our experiments were first performed using the laser to surface heat, immediately followed by (or overlapping with slightly) brief exposure to EM radiation $(10 \mathrm{~ms})$. The laser pulse was typically $6-10 \mathrm{~ms}$ in duration, which is not sufficient to ignite or cause self-heating in the HMX.

Figure 4 shows typical results from the experimental approach described above. After many ( $>50$ ) experiments, it was observed that the results fell into two categories: 1) (Figure 4 left) ambiguous results; is unclear whether ignition occurred due to continued self-heating after the termination of the laser pulse, or the EM pulse produced an actual breakdown, or 2) (Figure 4 right) results that may support our hypothesis; the surface is cooling after termination of the laser pulse and the ignition event occurs within the EM pulse. With this experimental approach, the EM enhanced ignition was difficult to consistently observe due to the rapid heating by the laser and subsequent nonlinear temperature-dependent nature of the ignition kinetics in the system (i.e. an "over-driven" condition).[18] The point at which the high activation energy processes take over is a very fine line in a high power laser-heated system, thus the heating rate induced by the laser was sufficiently high that we did not consistently isolate the EM enhanced ignition effect performing the experiments in this way.

We modified our approach by increasing the time elapsed between the end of the laser pulse and beginning of EM exposure (10 - $20 \mathrm{~ms}$ after termination of laser pulse). This strategy induced some degree of self-heating in the HMX due to energy absorption from the laser (which usually occurred once the laser pulse ended) that caused surface ignition but not sustained 
combustion. The surface cooled at slow enough rates, relative to the high heating rate of the original method, to systematically observe EM-induced plasma formation after the IR and visible light detector signals had both returned to baseline. Figure 5 shows a representative case of experiments that were run approx. 30 times. After the light caused by the laser heating subsided, the temperature probe returned to baseline (or $<80{ }^{\circ} \mathrm{C}$ as determined by prior calibration), and the $10 \mathrm{~ms}$ EM pulse was delivered. This EM pulse condition, on its own, will not induce any type of response in the sample (light generation, heating, etc). However as shown in Figure 5, delivery of the EM pulse at this point in the experiment results in plasma formation (very bright white light with much greater intensity than that generated by the laser alone) as well as a sharp increase in both the IR and visible light detector responses. Waiting a greater length of time after the end of the laser pulse to apply the EM pulse ensures that sufficient HMX decomposition intermediates have formed in the gas phase so that plasma formation will occur. We found this behavior to be reproducible between wait times of $9-17 \mathrm{~ms}$; the effect was again inconsistent if the time varied between the end of the laser pulse and start of the EM pulse was less than 9 ms or greater than $17 \mathrm{~ms}$.

To further illustrate the how the quantity of EM 'active' gas phase species develops after laser surface heating over time, a COMSOL model was run. The COMSOL multiphysics modeling platform incorporates the established kinetic model of HMX referred to above[17]; the details of the 4-step reversible kinetics and associated rate equations for HMX, and how they are used in COMSOL modeling, have been covered in previous publications [2,21]. In this case, the model was built for 1D surface heating, the laser pulse was applied for $13 \mathrm{~ms}$, and the reaction continues to grow up to $16 \mathrm{~ms}$, then dies out. Figure 6 shows the results of this model, with the mass fraction of species present in the gas phase above the sample surface plotted as a function of distance from the surface. Three results are given at different times, where $t=0$ is the beginning of the laser pulse. The total species population is the area under the curves, and this illustrates how the total population grows with time, including after the reaction has ceased. The model also reveals (not shown in plot) that the temperature of the surface is cooling at approx. $4 \mathrm{~K} / \mathrm{ms}$ just after the laser is turned off. Overall, these modeling results support our observations of EM-enhanced ignition (Figure 5) when the EM pulse occurs during the time in which sufficient decomposition intermediates are present in the gas phase in the volume space above the sample surface. Furthermore, this supports our approach of waiting several ms after the end of the laser pulse when the surface is cooling - with a larger volume of plasma precursor gas phase molecules present - to apply the EM pulse for observation of the plasma effect. Experimentally we showed that sufficient gas phase species were still present $17 \mathrm{~ms}$ after the end of the laser pulse. Note that there are numerous caveats and assumptions present in this numerical study; however, this model is meant to illustrate how the distribution of plasma precursor gas phase molecules changes with time. As such this representation may not be truly quantitative, but is conceptually accurate and agrees qualitatively with the models developed by Liau and Lyman.[18]

\section{Conclusion}

We have shown that the presence of an EM field will disrupt the thermal decomposition pathway of HMX, and perhaps other energetic materials with sufficient vapor pressure generation upon heating, and cause ignition to occur at a lower temperature than what is predicted under typical thermal conditions. While this study was not specifically designed to 
elucidate the exact mechanism, a likely scenario is that as heated HMX begins to sublime, charged decomposition intermediate species and free electrons in the gas phase interact strongly with EM energy and transfer additional non-thermal energy into the system, leading to plasma formation. Such an intense interaction would result in electrically excited molecules that propagate the energy and surpass the activation barrier for ignition. We isolated this effect in HMX using surface heating with a laser, followed by a "wait" period of $15-20 \mathrm{~ms}$ to allow for the formation of a sufficient volume of HMX decomposition intermediates in the gas phase, followed by a $10 \mathrm{~ms}$ EM pulse. The rapid absorption of EM energy during this coupling event is observed in the measured reflected power data. Ignition and plasma formation were monitored using both visible and IR photodiode probes, as well as imaged using a high-speed video camera. Our observation of EM interaction with energetic materials is a unique phenomenon because thermal decomposition is 'interrupted' by a plasma event before sustained combustion has begun to occur. This effect is distinct from plasma-assisted combustion and other ionization-coupled ignition scenarios because our process is interacting with the ions/radicals that result from the decomposition chemistry of HMX as opposed to combustion-induced ionization or coupling to a flame. Understanding the decomposition of energetic materials under the influence of EM energy is important to continuing to improve our understanding and predictive capabilities of material response under a variety of environmental conditions, and for the development of novel initiation techniques.

\section{Acknowledgements}

The authors wish to thank Bryan Henson, Laura Smilowitz, Bryce Tappan, John Yeager, and Genevieve Watt (LANL) for helpful discussions and experimental assistance. We also thank Jeffrey Kay (Sandia) for early insight. Los Alamos National Laboratory, an affirmative action equal opportunity employer, is operated by Los Alamos National Security, LLC, for the National Nuclear Security Administration of the U.S. Department of Energy under contract DE-AC5206NA25396. This work was supported by the Los Alamos Laboratory Directed Research and Development (LDRD) program, LA-UR-16-28948.

\section{References}

[1] W.L. Perry, T.D. Sewell, B.B. Glover, D.M. Dattelbaum, Electromagnetically induced localized ignition in secondary high explosives, J. Appl. Phys. 104 (2008) 094906.

[2] W.L. Perry, J.A. Gunderson, B.B. Glover, D.M. Dattelbaum, Electromagnetically induced localized ignition in secondary high explosives: Experiments and numerical verification, $\mathrm{J}$. Appl. Phys. 110 (2011) 034902.

[3] C.M. Tarver, Effect of electric fields on the reaction rates in shock initiating and detonating solid explosives, AIP Conference Proceedings 1426 (2012) pp. 227-230.

[4] M.E. Daily, B.B. Glover, S.F. Son, L.J. Groven, X-Band Microwave Properties and Ignition Predictions of Neat Explosives, Propellants, Explos., Pyrotech. 38 (2013) 810-817.

[5] W.L. Perry, A.L. Higginbotham Duque, Micro to mesoscale temperature gradients in microwave heated energetic materials, J. Appl. Phys. 116 (2014) 054911.

[6] W.J.M. Kort-Kamp, N.L. Cordes, A. Ionita, B.B. Glover, A.L.H. Duque, W.L. Perry, B.M. 
Patterson, D.A.R. Dalvit, D.S. Moore, Microscale Electromagnetic Heating in Heterogeneous Energetic Materials Based on X-ray Computed Tomography, Phys. Rev. Applied 5 (2016) 044008.

[7] B. Wolk, A. DeFilippo, J.Y. Chen, R. Dibble, A. Nishiyama, Y. Ikeda, Enhancement of flame development by microwave-assisted spark ignition in constant volume combustion chamber, Combust. Flame 160 (2013) 1225-1234.

[8] A. Starikovskiy, Physics and chemistry of plasma-assisted combustion, Phil. Trans. R. Soc. A 373 (2015) 20150074.

[9] A.L. Duque, W.L. Perry, Microwave-Induced Heating of HMX for Decomposition Kinetic Analysis, MRS Proceedings 1405 (2012) doi: 10.1557/opl.2012.105.

[10] A.L. Duque, W.L. Perry, Microwave-Induced Fast Thermal Decomposition of HMX, Fifteenth International Detonation Symposium, Office of Naval Research, Arlington, VA (2014), pp. 154-162.

[11] B.F. Henson, B.W. Asay, L.B. Smilowitz, P.M. Dickson, Ignition chemistry in HMX from thermal explosion to detonation, AIP Conference Proceedings 620 (2002) pp. 1069-1072.

[12] P.M. Dickson, B.W. Asay, B.F. Henson, L.B. Smilowitz, Thermal cook-off response of confined PBX 9501, Proc. R. Soc. Lond. A 460 (2004) pp. 3447-3455.

[13] L. Smilowitz, B.F. Henson, J.J. Romero, B.W. Asay, C.L. Schwartz, A. Saunders, F.E. Merrill, C.L. Morris, K. Kwiatkowski, G. Hogan, P. Nedrow, M.M. Murray, T.N. Thompson, W. McNeil, P. Rightley, M. Marr-Lyon, Direct observation of the phenomenology of a solid thermal explosion using time-resolved proton radiography, Phys. Rev. Lett. 100 (2008) 228301.

[14] B.F. Henson, L. Smilowitz, J.J. Romero, B.W. Asay, Modeling Thermal Ignition and the Initial Conditions for Internal Burning in PBX 9501, AIP Conference Proceedings 1195 (2009) pp. 257-262.

[15] B.F. Henson, L.B. Smilowitz, The Chemical Kinetics of Solid Thermal Explosions, in: B.W. Asay (Ed.), Non-Shock Initiation of Explosives, Springer, New York, 2010, pp. 48-128.

[16] R. Behrens. Thermal Decomposition of HMX: Morphological and Chemical Changes Induced at Slow Decomposition Kinetics. Twelfth International Detonation Symposium, Office of Naval Research, Arlington, VA (2002), pp. 519-529.

[17] P.M. Dickson, B.W. Asay, B.F. Henson, C.S. Fugard, J. Wong, Measurement of phase change and thermal decomposition kinetics during cookoff of PBX 9501, AIP Conference Proceedings 505 (2000) pp. 837-840.

[18] Y.C. Liau, J.L. Lyman, Modeling laser-induced ignition of nitramine propellants with condensed and gas-phase absorption, Combust. Sci. Technol. 174 (2002) 141-171.

[19] C.M. Tarver, Chemical Kinetic Modeling of HMX and TATB Laser Ignition Tests, J. Energ. Mater. 22 (2004) 93-107.

[20] A.N. Ali, S.F. Son, B.W. Asay, M.E. Decroix, M.Q. Brewster, High-irradiance laser ignition of explosives, Combust. Sci. Technol. 175 (2003) 1551-1571.

[21] W.L. Perry, J.A. Gunderson, M.M. Balkey, P.M. Dickson, Impact-induced friction ignition of an explosive: Infrared observations and modeling, J. Appl. Phys. 108 (2010) 084902. 


\section{General Ensemble Stages of HMX Thermal Decomposition}

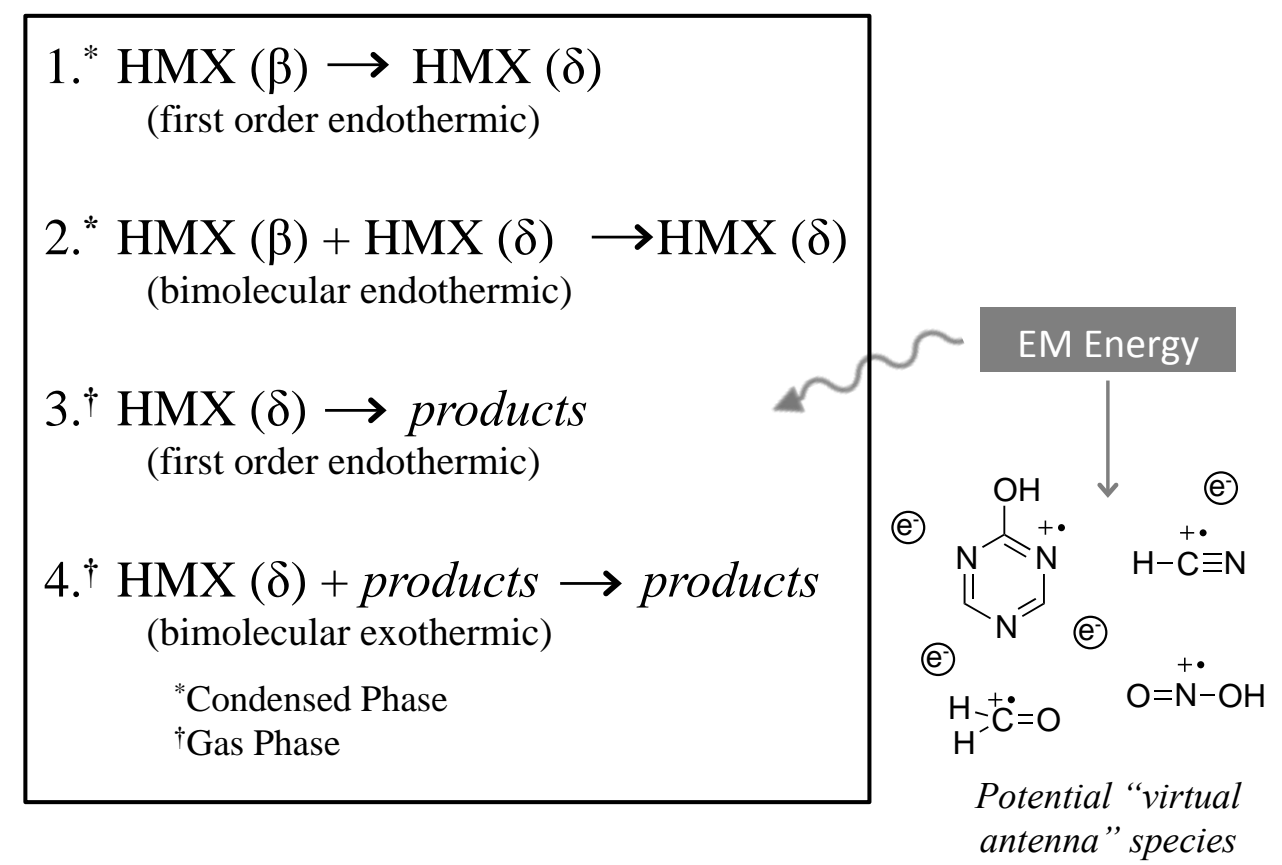

Figure 1. A general four-step kinetic pathway for the thermal decomposition of HMX is shown in the box. [17] We hypothesize that once thermal decomposition intermediate species and free electrons are present in the gas phase (Step 3), the introduction of EM energy will effect the typical progression of Step 4 by providing additional nonthermal energy into the system. The molecular structures shown outside the box are some of the potential ionized decomposition intermediates present in the gas phase that will undergo enhanced reactivity upon application of EM energy; these are examples of "virtual antennas" because their presence will cause preferential interaction with EM energy.

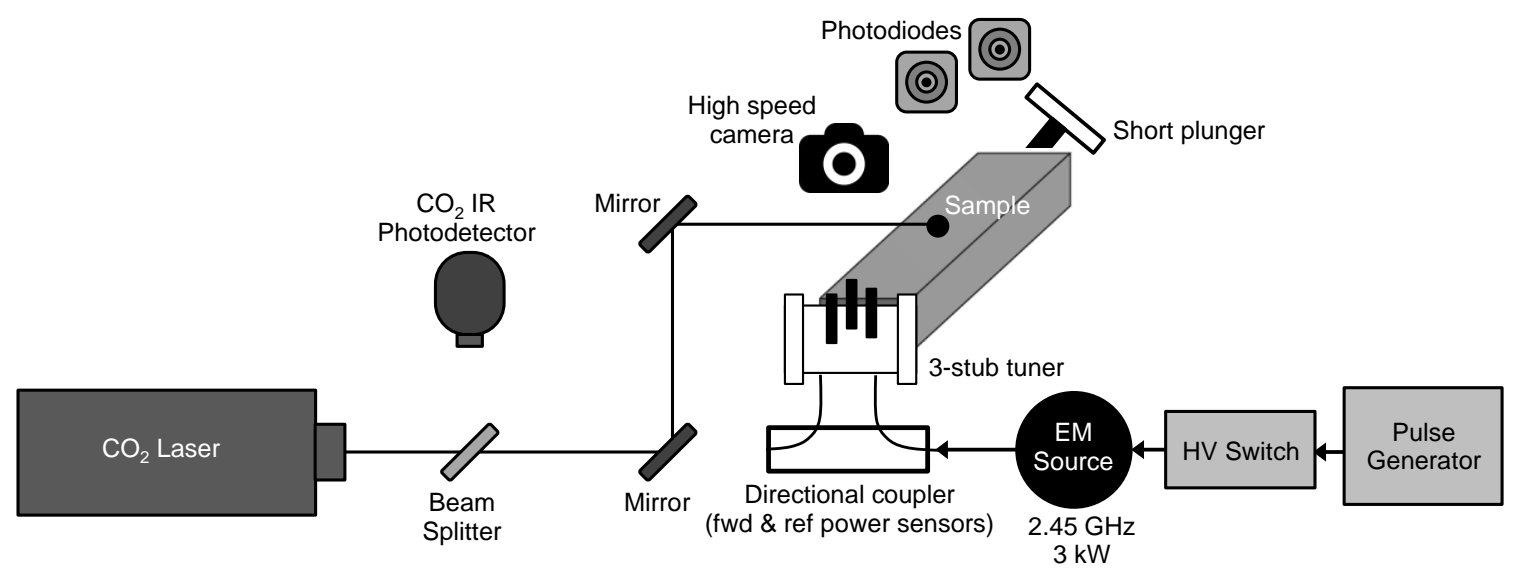

Figure 2. Schematic of laser heating experimental setup. 

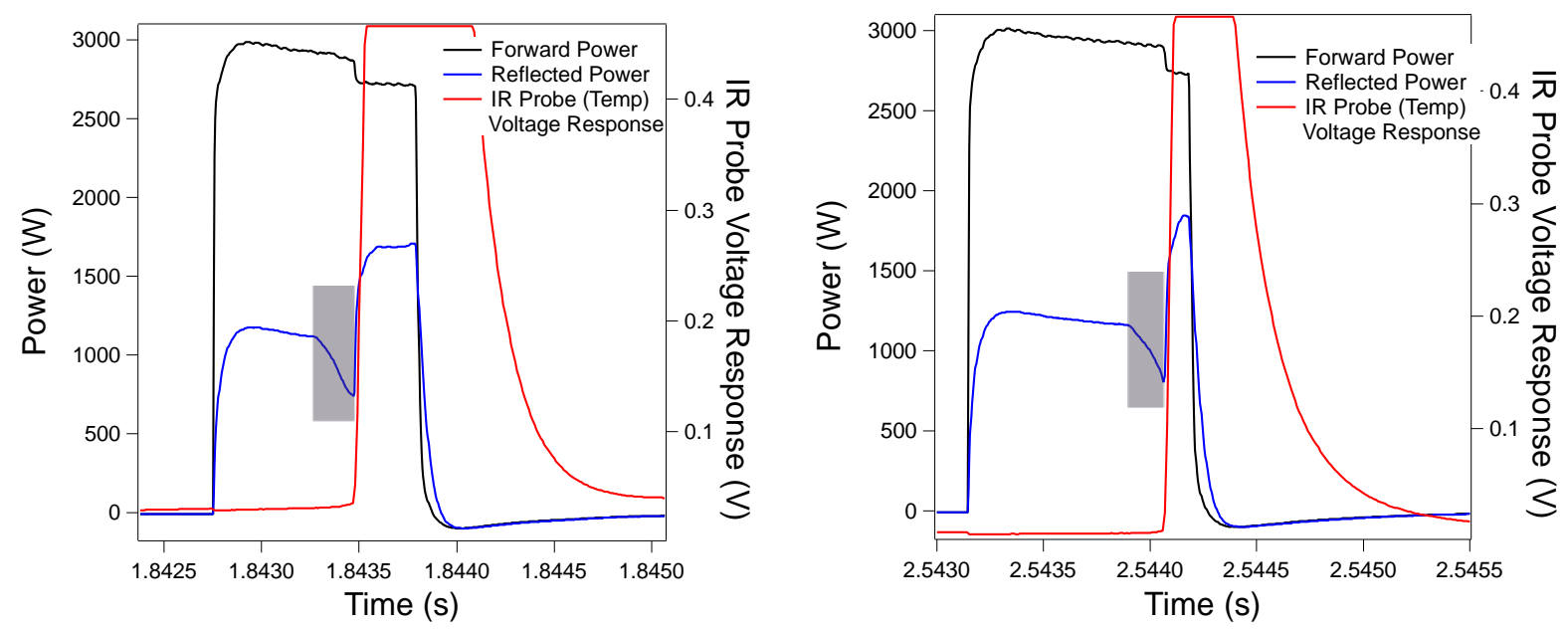

Figure 3. Data regions of two different EM pulses (1 ms duration each) during an experiment with HMX previously reported [10]. Highlighted in the two figures are regions in which the reflected power abruptly decreases just before an ignition event is observed, potentially representing the timing of enhanced coupling between the EM energy and gas phase ions/radicals.
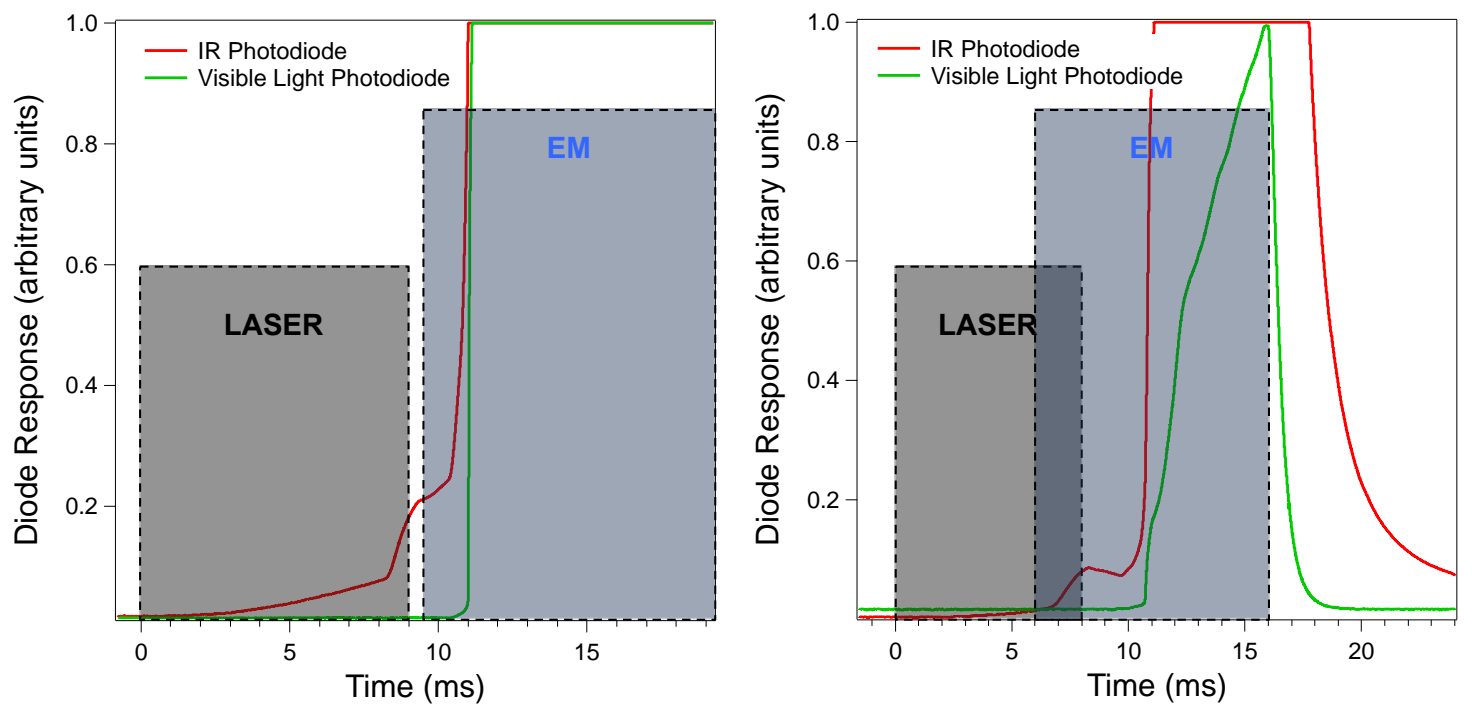

Figure 4. Two representative examples of the first experimental approach. The left figure shows ambiguous results; is unclear whether ignition occurred due to continued self-heating after the termination of the laser pulse, or the EM pulse produced an actual breakdown. The right figure shows results that may support our hypothesis; the surface is cooling after termination of the laser pulse and the ignition event occurs within the EM. 


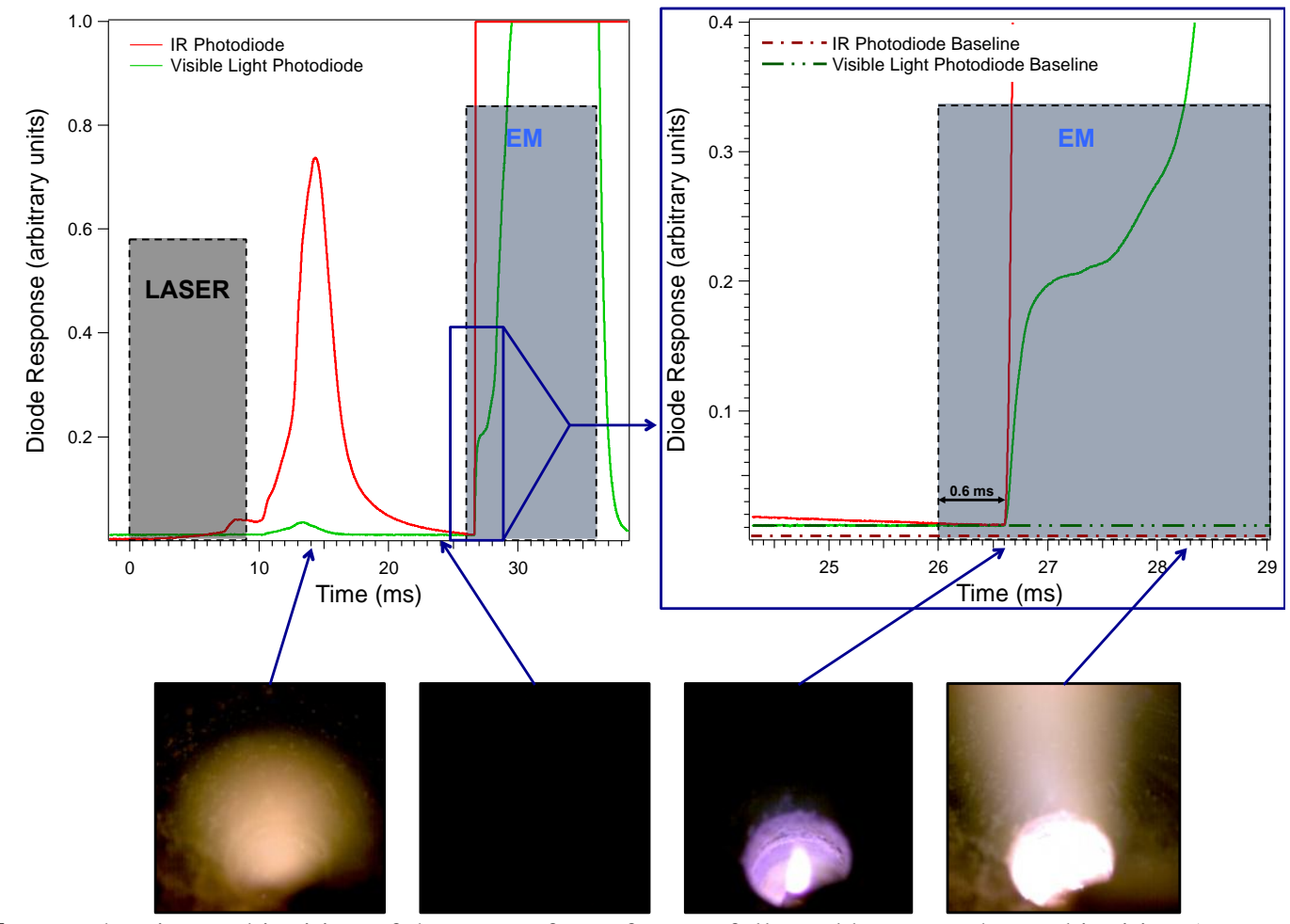

Figure 5. Laser heating and ignition of the top surface of HMX followed by EM enhanced ignition $17 \mathrm{~ms}$ after the end of the laser pulse. Both the visible and IR detector signals show that the sample was cooling and no flame from self-heating was present when the EM exposure began, and plasma formation occurred <1 ms later. The images at the bottom were taken with high-speed video and illustrate the differences in color and intensity of the flames.

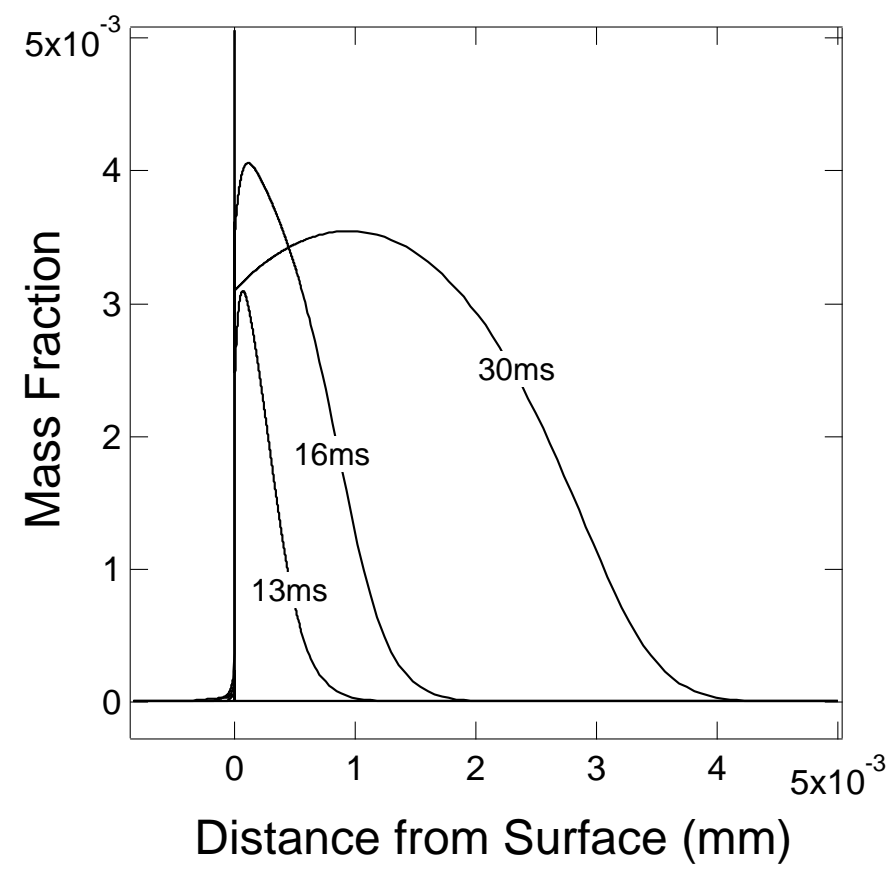

Figure 6. COMSOL model of 1-D surface heating of HMX. The mass fraction is that of the decomposition intermediates present in the gas phase above the sample surface. The total species population is the area under the 
curves, therefore the total population grows with time, including after both the laser pulse and subsequent reactions have ceased (after 13 and $16 \mathrm{~ms}$, respectively). 